\title{
Head movement assessment of cerebral palsy users with severe motor disorders when they control a computer thought eye movements
}

\author{
A. Clemotte, M.A. Velasco, E. Rocon \\ erocon@csic.es \\ CAR UPM-CSIC, Ctra. Campo Real, km 0.2, 28500, Arganda del Rey, Spain
}

\begin{abstract}
Eye tracking is currently a promising technology to access computers for people who suffer severe motor disorders, like cerebral palsy. However, there is a lack of usability assessment procedures and concrete value to describe the user's motor capabilities in this specific scenario of computer control. This paper presents a methodology, based on two head movement assessment metrics and the ISO-9241, for the quantitative motor description of users with severe motor disorders, when they control the computer thought their eyes. Seven participants with $C P$ and three people without motor disabilities were recruited for the evaluation of the proposed procedure. Results evidence for the first time how users with CP control their head while they access a computer with their eyes.
\end{abstract}

Keywords: Usability, Motor impairments, Cerebral palsy, Pointing task, Inertial sensors, Eye tracking.

\section{INTRODUCTION}

"Cerebral palsy (CP) describes a group of disorders of the development of movement and posture, causing activity limitation, which are attributed to nonprogressive disturbances that occurred in the developing fetal or infant brain. The motor disorders of cerebral palsy are often accompanied by disturbances of sensation, cognition, communication, perception, and/or behaviour, and/or by a seizure disorder" [1]. CP limits motor and postural control, which reduce their abilities to control the computers through commonly used interfaces: mouse, keyboard or touchscreen.

There are studies aimed at developing innovative alternative interfaces in response to the special needs of people with motor disorders. An alternative interface is an input interface which can be connected to electronic devices (computers, tablets, smart phones) allowing users to input data using physical gestures (pointing, clicking or dragging) or by other alternative commands (voice, evoking potential motor cortex, etc.) for voluntary actions.
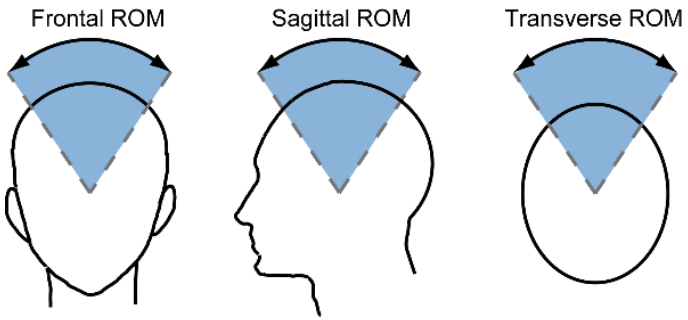

Figure 1. The abnormal movements and postures can be identified by measuring the Range of Motion (ROM) of the head, in the three orthogonal planes.

There are also software applications which provide support to these interfaces, decreasing the effects of certain involuntary movements, through clicking alternative techniques [2], filter techniques [3][4] among others.

Eye tracking systems are widely used by people with cerebral palsy in Spain, especially by the ASPACE Confederation, which groupes almost 18000 people with cerebral palsy. However, some users with CP cannot control this interface, possibly due to their low cervical control capabilities.

This paper aims to propose a procedure to measure the head motor characteristics of people with $\mathrm{CP}$ with severe motor disorders when they control the computer thought an eye tracking system. The assessment methodology is based in two metrics: head range of movement and head peak frequency, in the three anatomical planes of the head: frontal, sagittal, and transversal. The overall process was evaluated with the ISO 9241 standard, in order to produce rigorous and comparable results. In addition, we evaluated the methodology and leave precedent of the motor characteristics of users severely affected by the PC.

We expect these measures to be able to serve as guidelines to establish design requirements for new developments around the eye-trackers, specially designed for people with cerebral palsy with severe motor disorders. 


\section{MOTOR ASSESSMENT METRICS}

\subsection{HEAD RANGE OF MOTION (ROM)}

The abnormal movements and postures can be identified by measuring spatial variables, such as the head range of motion. Cervical movements are clinically described as rotations around three orthogonal axes which can be defined mathematically by Euler formulation, which defines rotation on three axes: frontal, sagittal and transverse. The ROM is defined as the difference between maximum and minimum values of the Euler angles. A graphical representation of the ROM in the frontal, sagittal, and transverse planes is shown in Figure 1.

\subsection{HEAD PEAK FREQUENCY (HPF)}

An analysis in the frequency domain of the head movement was also performed. This analysis is necessary because the motor alterations may vary in frequency. For our analysis, we calculated the frequency at which there is the greatest spectral density of the head movement in all three axes of rotation.

\subsection{ISO 9241}

The ISO 9241-9 "Ergonomic requirements for office work with visual display terminals (VDTs) - Part 9: Requirements for non-keyboard input devices" [5] provides guidelines and assessment procedures for testing computing devices in reaching target exercises. The ISO is based on Fitts's law [6] which models human psychomotricity behavior based on Shannon's theorem. This model stablishes an index to define the effort of a reaching target task, the "index of difficulty", based in two parameters: the target diameter (W) and the amplitude of the movement (A), (1). By means of this model, we can parameterize and limit the reaching target task on the screen.

$$
I D=\log _{2}\left(\frac{A}{W}+1\right)
$$

\section{METHODOLOGY}

The test consisted in a reaching target task, performed through an eye-tracker system, bounded by the ISO 9241 standard, while a head tracking system measured the orientation of the head.

\subsection{INSTRUMENTS}

\subsubsection{Eye-tracker (ET)}

There are several type of eye-trackers. The less invasive ones are those based in video oculography. Among these, the ones based on infrared light (IR) are preferred due to their robustness and stability.

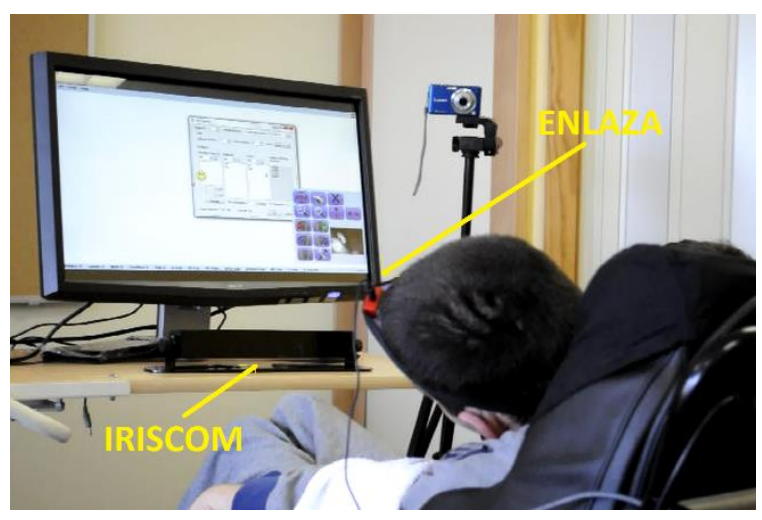

Figure 2. The aim of this study was to assess the movement of the head while severely affected users with motor disorders control the computer through their eyes. THe figure illustrate a moment during the trial: the user's back, the eye-tracker and the headtracker.

IR eye-trackers consists of two infrared light sources, which illuminate the eyes of the user and provide benchmarks to the tracking system. The IR produced must have a wavelength of $800 \mathrm{~nm}$ (found naturally in sunlight and incandescent lamps) and the power of the emitters should by according to the safety guidelines of the ACGIH. Besides the infrared light source, an infrared camera captures the reflected light. The infrared camera provides an image within a fixed horizontal band. Specific software interprets this image, calculates where the user is looking, and translates that position into new coordinates for the mouse pointer.

Eye-trackers allows the users to control a computer by his eye movements, substituting the standard mouse. Based on these criteria we have chosen the PRIMMA eye-tracker (IRISBOND, Spain).

\subsubsection{Head-tracker (HT)}

The movement of the head can be recorded with inertial measurement unit (IMU) systems. The IMU integrates a three-axis gyroscope, accelerometer and magnetometer.

The ENLAZA device complies with these requirements. It consists of a commercial helmet and an inertial measurement unit (IMU) [7]. This inertial 
sensor was developed to measure kinematic parameters to describe pathologic patterns.

\subsection{EXPERIMENTAL SETUP AND PROCEDURE}

In the experiments, the eye-tracker system controlled the mouse pointer while the head-tracker measured the posture of the head. An automatic selection (click) was performed if the user kept the cursor within an area of $2 \mathrm{~cm}$ a time of $1 \mathrm{~s}$. These area and time were defined by the occupational therapists, who had experience in the use of alternative communication system.

The participants were asked to perform reaching target tasks. They had to locate the cursor over the target as fast as possible by moving the eyes and then select it. All targets were displayed at the beginning of the test. The target that should be selected by the participant turned blue while the others remained gray, Figure 3 . The software FittsStudy [8] was used to control the target position.

The indexes of difficulty (ID) of the tasks were defined by the ISO 9241-9, which depends on the: target diameter and movement amplitude. The values of the parameters of the tasks that the participant performed were: target diameter 75-100 pixels and movement amplitude $275-350$ pixels.

The tasks were displayed on a monitor with a vertical resolution equal to 768 pixels and 1366 pixels of horizontal resolution. Figure 2 depicts a moment during the test.

Before the beginning of the test, a proper placement of the participants in front of the computer screen was granted. An occupational therapist was in charge of the position and posture of the users. The OT was asked to place the users in the vertical plane perpendicular to the center of the screen, at a distance between 60 and $80 \mathrm{~cm}$. The OT made sure that the users were in the center of the eye tracker's track box before starting the test.

It took about 40 minutes per subject to complete a test. The task was interrupted if the user was feeling very tired or unmotivated, returning to resume the task after a moment, if possible.

\subsection{PARTICIPANTS}

Seven people with CP with severe motor disorders and three users without disabilities (acting as a control group) were recruited. The mean and standard deviation of the participants' ages are 29 and 6, respectively. The tests were carried out in ASPACE Cantabria (Santander, Spain). ASPACE Cantabria therapists are experienced in the use of alternative devices such as the eye tracking interfaces.

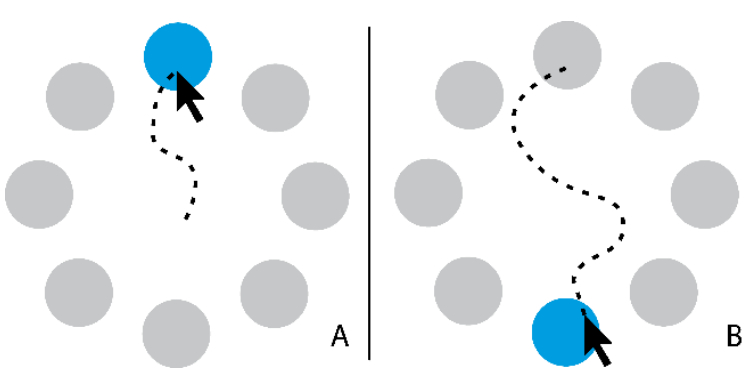

Figure 3. Subjects were asked to perform reaching target tasks, described by the index of difficulty defined by the ISO-9241. Participants locate the cursor over the target as fast as possible by moving the eyes and then select it.

Table. 1. Participants recruited with its age and clinical motor control descriptors. All the participants with CP are seriously affected by motor impairments.

\begin{tabular}{|l|r|l|l|}
\hline Subject & \multicolumn{1}{|c|}{ Age } & $\begin{array}{l}\text { GMFCS / } \\
\text { MACS }\end{array}$ & Cervical tone \\
\hline CP1 & 28 & $5 / 5$ & Hypertonia \\
CP2 & 31 & $4 / 4$ & Hypotonia \\
CP3 & 37 & $4 / 4$ & Hypotonia \\
CP4 & 28 & $5 / 5$ & Hypertonia \\
CP5 & 22 & $4 / 5$ & Hypotonia \\
CP6 & 22 & $5 / 5$ & Hypotonia \\
CP7 & 36 & $5 / 4$ & Hypertonia \\
HP1 & 26 & - & - \\
HP2 & 33 & - & - \\
HP3 & 35 & - & - \\
\hline
\end{tabular}

Deficits in trunk control affect the stability of the head as pointed out by Saavedra [10]. Consequently, pelvic and torso support was provided for those participants with poor trunk postural control.

All participants were men. None suffers from a severe intellectual disability. All the participants with $\mathrm{CP}$ used a wheelchair and had assistance requirements. They were classified as GMFCS between 4 and 5 [9]. In addition, all the users with CP had incremented or decremented muscle tone, which was described with the terms of Hypertonia and Hypotonia. Hypertonia is increased muscle tone, and lack of flexibility and Hypotonia otherwise. Table 1 describes the profile of the users. It is important to emphasize the severity of the motor disorder of the impaired group. 


\section{EXPERIMENTAL RESULTS}

Both groups of users completed 1053 reaching target tasks. After data preprocessing, 679 of them were chosen: 475 from users with CP and 204 from users without disabilities. The rest were discarded due to a loss of synchronization between the devices or due to inconsistent measures.

Figure 4 shows the distribution of the error rate per participant. Data indicates that the CP participants had $59 \%$ more error rate than participants without disabilities, $45 \%$ and $27 \%$ respectively. The subjects distinguished by its error rate were CP3 and HP1, for their wide dispersion, relative to other participants.

\subsection{HEAD RANGE OF MOTION (ROM)}

The ROM values of the participants with $\mathrm{CP}$ were widely scattered. CP2 had the highest ROM in the frontal plane $\left(82^{\circ}\right)$ and $\mathrm{CP} 6$ had the lowest ROM value in the sagittal plane $\left(2^{\circ}\right)$. Both users were diagnosed with hypotonic tone.

There is not a relationship between the ROM of users with $\mathrm{CP}$ with the same tone (hypotonic or hypertonic). For example, the differences between the averages ROM of CP5 and CP6, who have a hypotonic tone, were $3^{\circ}$. However, there is a difference of $57^{\circ}$ between $\mathrm{CP} 2$ and $\mathrm{CP} 5$, both of them with hypotonic tone also. The ROM of the users with hypertonic CP (CP1 and CP7) were higher than the ROM of users with hypotonic CP (CP3, CP5 and CP7), as was expected, except for the case of $\mathrm{CP} 2$.

Some users with CP have a ROM similar to the ROM of the user without disabilities. This is the case of CP5 (with hypotonic tone), who has an average ROM similar to the average ROM of HP.

The frontal plane of the users with cerebral palsy and had the highest ROM value, followed by the transversal and sagittal.

ROM is related to the ability to maintain posture, which has a direct involvement in the eye-tracker measurement. We concluded that users with CP have greater ROM value than users without disabilities.

Table 2 summarizes the metrics presented, for the participants with $\mathrm{CP}$ and without disabilities. It depicts the mean and standard deviation value for each case.

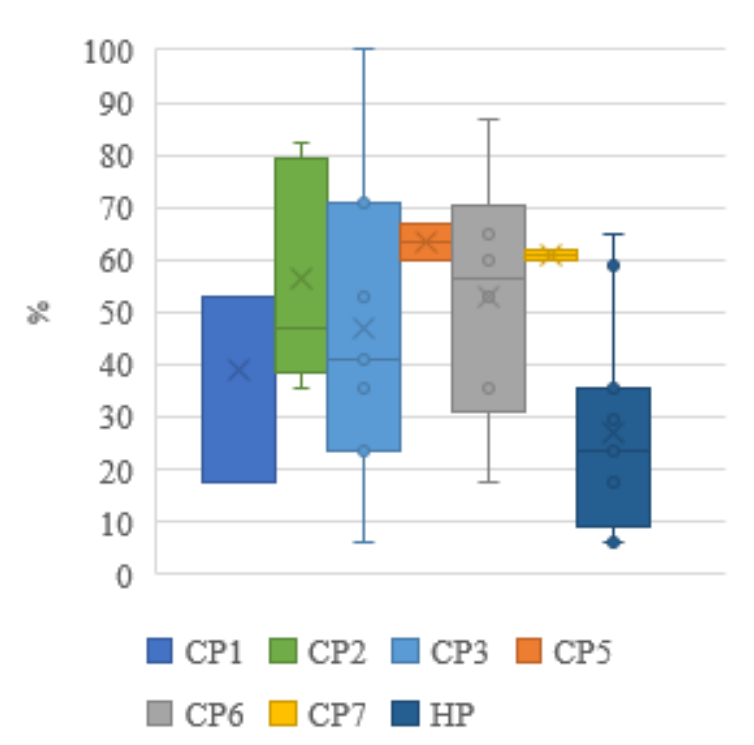

Figure 4. Mean error rate (\%) of each user with its standard deviation.

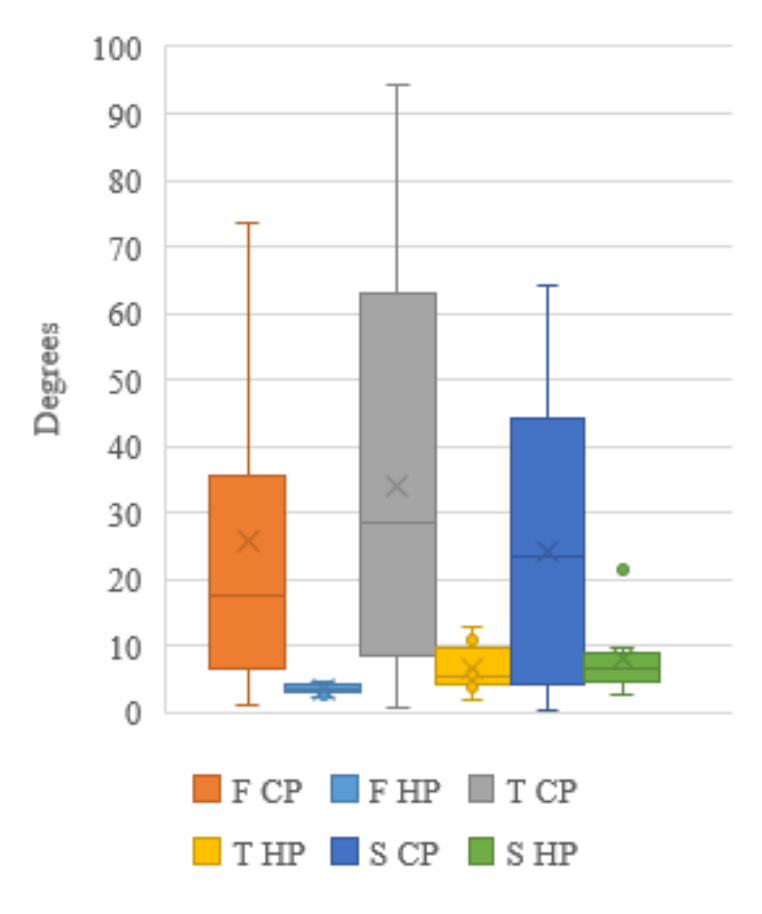

Figure 5. Mean $\operatorname{ROM}\left({ }^{\circ}\right)$ of each user with its standard deviation, from each anatomical plane: frontal $(\mathrm{F})$, transversal (T), and sagittal (S).

\subsection{HEAD PEAK FREQUENCY (HPF)}

Unlike the ROM, which had the highest value in the transversal plane, the head peak frequency was highest in the sagittal plane. 


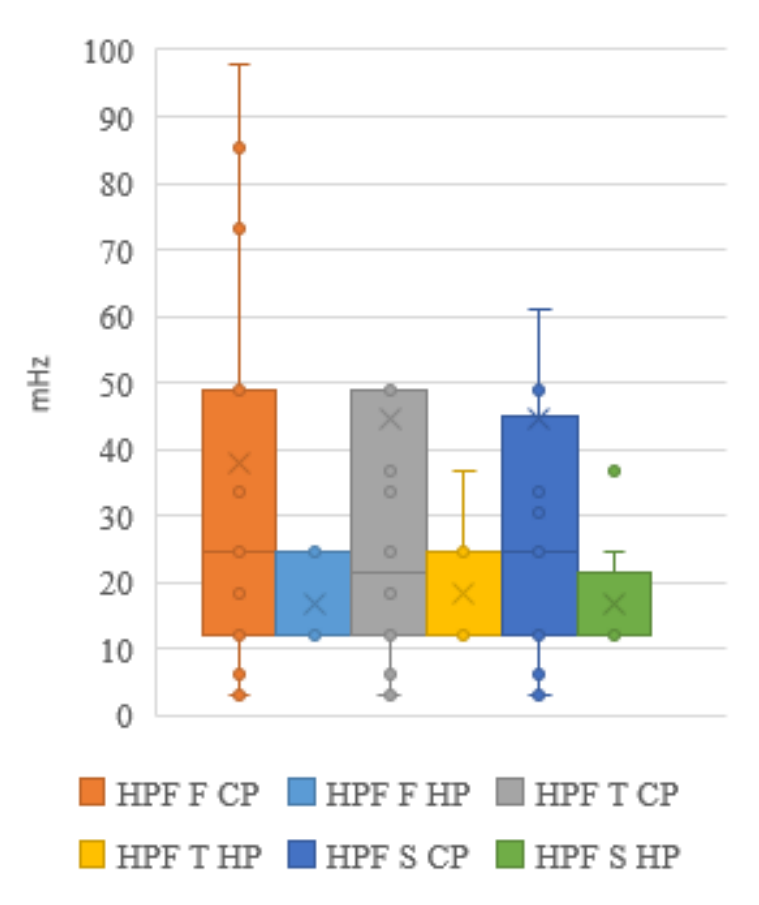

Figure 6. Mean head peak frequency $(\mathrm{mHz})$ of each user, with its standard deviation, from each anatomical plane.

Involuntary movements from users with $\mathrm{CP}$ had higher frequency components than the voluntary movements from users without disabilities. The HPF measured in users with $\mathrm{CP}$ was approximately two times the HPF estimated in users without disabilities.

Unlike what happened with the ROM, where the tone of the participant was reflected in the ROM, the HPF value between the users with $\mathrm{CP}$ is less scattered.

Most of the users with hypertonia had a greater HPF than the users with Hypotonia.

\section{DISCUSSION}

The aim of this paper was to stablish an assessment method for the evaluation of cervical motor characteristics while people with severe motor disabilities controlled a computer through their eyes. In addition, we evaluated our method with users with CP.

Eye tracking systems require to keep the head in a stable position inside its track box. Many users with $\mathrm{CP}$ are not able to do this, due to their poor cervical control. This limits the use of the eye tracker, reducing the performance metrics.

We measured the maximum head rotation range in the three defined planes and we found that its value in the frontal plane was $34^{\circ}, 39^{\circ}$ in the transversal, and in $28^{\circ}$ in the sagittal. In a study presented by Raya et al, they measured the ROM while users severely affected by motor disorders controlled a computer thought their head movements [11]. They measured a ROM value of $25^{\circ}$ in the frontal plane, $26^{\circ}$ in the sagittal and $32^{\circ}$ in the transversal. We argued that due the head based control of their study; the users maintained their head within a more closed range, unlike our control paradigm, which does not require the user any kind of control of the head.

Frontal, transverse and sagittal head range of motion were highly variable among all participants with $\mathrm{CP}$. High ROM value indicates that users with CP suffer from limitations in maintaining the posture, an important feature for the design of an appropriate interface. There is not a strong relation between ROM and the profile of participants with $\mathrm{CP}$ (hypertonic/hypotonic). Comparatively, it can be concluded that the users with $\mathrm{CP}$ generally have greater ROM than users without disabilities, in the order of $40^{\circ}$ in the transversal plane, $34^{\circ}$ in the frontal and 28 in the sagittal.

Table 2. Mean and standard deviation of each metric for each group of users.

\begin{tabular}{|l|l|l|l|}
\hline Metrics & CP & HP & Unit \\
\hline ERR & $45 \pm 25$ & $27 \pm 19$ & $\%$ \\
ROM F & $34 \pm 24$ & $3 \pm 1$ & degree \\
ROM T & $40 \pm 31$ & $6 \pm 4$ & degree \\
ROM S & $29 \pm 22$ & $8 \pm 6$ & degree \\
HPF F & $38 \pm 42$ & $17 \pm 6$ & $\mathrm{mHz}$ \\
HPF T & $41 \pm 55$ & $18 \pm 9$ & $\mathrm{mHz}$ \\
HPF S & $44 \pm 66$ & $17 \pm 9$ & $\mathrm{mHz}$ \\
\hline
\end{tabular}

The head peak frequency from users with $\mathrm{CP}$ was in the order of $40 \mathrm{mHz}$ for all the planes, approximately two times bigger than the peak from users without disabilities. Velasco et al measured the head movement, but when user with $\mathrm{CP}$ control the computers by this gesture [12]. They also measured the head peak frequency, reporting the following values: $580 \mathrm{mHz}, 540 \mathrm{mHz}$, and $680 \mathrm{mHz}$ for the frontal, sagittal, and transversal planes. These values are one order of magnitude higher than the values that we have measured. The main reason of this difference could be the use of the head movement to control the cursor on the screen.

\section{CONCLUSION}

We presented a methodology and metrics for the assessment of head movements while user with cerebral palsy severely affected with motor disorders controlled a computer with their eyes. 
The methodology is based in the standard ISO 9241-9 and two specially defined metrics, related to the head movements, in order to provided rigorous and comparable results: the head range of motion and the head peak frequency.

Experimental results suggest that users with $\mathrm{CP}$ had a head movement range of motion of $28^{\circ}$ to $39^{\circ}$, and a head peak frequency of $40 \mathrm{mHz}$. All values were greater than the values measured from users without disabilities, mainly because they had better control than subjects with $\mathrm{CP}$.

Knowing the characteristics of the users' head movements is essential, since posture and control limitation of people with CP decreased the eyetrackers performance. The head motor descriptors we measured must be used as a design requirement, so as to meet the inherent requirements of these complex users. This is also relevant for the HCI community, since there is lack of scientific works based on quantitative methodologies to assess the motor behavior of persons with severe motor disorders.

\section{Acknowledgments}

This work was possible thank to the projects NetMD (RTC-2015-3967-1), NeuroMOD (DPI2015-68664C4-1-R), and InterAAC (RTC-2015-4327-1). They are all financed by the Spanish Ministry of Economy, Industry and Competitiveness. Special thanks to ASPACE-Cantabria team, especially to T. Gonzalez and A. Ruiz.

\section{Bibliography}

[1] M. Bax, M. Goldstein, P. Rosenbaum, A. Leviton, N. Paneth, B. Dan, B. Jacobsson, and D. Damiano, "Proposed definition and classification of cerebral palsy," Dev. Med. child Neurol., vol. 47, no. 8, pp. 571-576, Jul. 2005.

[2] J. O. Wobbrock and K. Gajos, “A comparison of area pointing and goal crossing for people with and without motor impairments," in Proceedings of the 9th international ACM SIGACCESS conference on Computers and accessibility, 2007, pp. 3-10.

[3] J. O. Wobbrock, J. Fogarty, S. Liu, S. Kimuro, and S. Harada, "The angle mouse: targetagnostic dynamic gain adjustment based on angular deviation," in SIGCHI Conference on Human Factors in Computing Systems, 2009, pp. 1401-1410.

[4] R. Raya, E. Rocon, J. a Gallego, R. Ceres, and J. L. Pons, "A robust Kalman algorithm to facilitate human-computer interaction for people with cerebral palsy, using a new interface based on inertial sensors," Sensors, vol. 12, no. 3, pp. 3049-3067, Jan. 2012.
[5] "Ergonomic requirements for office work with visual display terminals (VDTs) - Part 9: Requirements for non-keyboard input devices ISO/DIS 9241-9.”.

[6] P. M. Fitts, "The information capacity of the human motor system in controlling the amplitude of movement. 1954.," J. Exp. Psychol. Gen., vol. 121, no. 3, pp. 262-269, Sep. 1992.

[7] R. Raya, E. Rocon, R. Ceres, and M. Pajaro, "A mobile robot controlled by an adaptive inertial interface for children with physical and cognitive disorders," in Technologies for Practical Robot Applications (TePRA), 2012 IEEE International Conference on, 2012, pp. 151-156.

[8] J. O. Wobbrock, K. Shinohara, and A. Jansen, "The effects of task dimensionality, endpoint deviation, throughput calculation, and experiment design on pointing measures and models," in Proceedings of the 2011 annual conference on Human factors in computing systems - CHI '11, 2011, p. 1639.

[9] R. Palisano, P. Rosenbaum, S. Walter, D. Russell, E. Wood, and B. Galuppi, "Development and reliability of a system to classify gross motor function in children with cerebral palsy.," Dev. Med. child Neurol., vol. 39, no. 4, pp. 214-23, Apr. 1997.

[10] S. Saavedra, M. Woollacott, and P. Van Donkelaar, "Head stability during quiet sitting in children with cerebral palsy: Effect of vision and trunk support," Exp. Brain Res., vol. 201, pp. 1323, 2010.

[11] R. Raya, E. Rocon, R. Ceres, J. M. Belda, and J. Laparra, "Positive and negative motor signs of head motion in cerebral palsy an analysis based on a wearable inertial human computer interface," ISSNIP Biosignals Biorobotics Conf. Biosignals Robot. Better Safer Living, pp. 1-5, Feb. 2013.

[12] M. A. Velasco, R. Raya, R. Ceres, A. Clemotte, A. Ruiz, and T. González, "Positive and Negative Signs of Head Motion in Cerebral Palsy: Assessment of Impairment and Task Performance.," IEEE Syst. J. Manuscr., vol. 8, no. $0,2014$. 